\title{
Comparison of CTA to DSA in Determining the Etiology of Spontaneous ICH
}

\author{
Robert Yeung, Tabassum Ahmad, Richard I. Aviv, Lyne Noel de Tilly, \\ Allan J. Fox, Sean P. Symons
}

\begin{abstract}
Background and Purpose: To compare the efficacy of computed tomographic angiography (CTA) to that of digital subtraction angiography (DSA) in the detection of secondary causes of intracerebral hemorrhage (ICH). Methods: Between January 2001 and February 2007 there were 286 patients that had both CTA and DSA for intracranial hemorrhage of all types. Those with primarily subarachnoid hemorrhage or recent trauma were excluded. Fifty-five patients formed the study cohort. Three reviewers independently analyzed the CTAs in a blinded protocol and classified them based on presence or absence of a secondary etiology. Results were compared with the reference standard DSA and kappa values determined for interobserver variability. Results: The overall sensitivity, specificity, positive predictive value, negative predictive value and accuracy of CTA were $89 \%, 92 \%, 91 \%, 91 \%$ and $91 \%$, respectively. Kappa value for interobserver agreement ranged from 0.78 to 0.89 . Two of four dural arteriovenous fistulas (dAVF) were missed on CTA by all three reviewers. Conclusion: CTA is nearly as effective as DSA at determining the cause of secondary intracerebral hemorrhage, but with a lower sensitivity for dAVFs. This supports the use of CTA as the first screening test in patients presenting with spontaneous ICH.
\end{abstract}

RÉSUMÉ: Comparaison de l'ACT à l'ASD pour déterminer l'étiologie de l'HIC spontanée. Contexte et objectif : Notre but était de comparer l'efficacité de l'angiographie par tomodensitométrie (ACT) à l'angiographie de soustraction digitale (ASD) dans la détection de causes secondaires de d'hémorragies intracérébrales (HIC). Méthodes : Entre janvier 2001 et février 2007, 286 patients ont subi une ACT et une ASD pour différents types d'HIC. Les patients atteints d'une hémorragie sous-arachnoïdienne primaire ou d'un traumatisme récent ont été exclus. Cinquante-cinq patients ont été inclus dans l'étude. Trois réviseurs ont analysé les ACT de façon indépendante et à l'aveugle et les ont classifiées quant à la présence ou à l'absence d'une étiologie secondaire. Les résultats ont été comparés à l'ASD comme standard de référence et la variabilité interobservateur a été estimée au moyen du coefficient kappa. Résultats : La sensibilité, la spécificité, la valeur prédictive positive, la valeur prédictive négative et l'exactitude de l'ACT étaient respectivement de $89 \%, 92 \%, 91 \%, 91 \%$ et $91 \%$. Le coefficient kappa pour la concordance interobservateur était de 0,78 à 0,89 . Deux sur quatre fistules durales artério-veineuses ont été manquées à l'ACT par les trois réviseurs. Conclusion : L'ACT est presque aussi efficace que l'ASD pour déterminer la cause d'une hémorragie intracérébrale secondaire, mais elle est moins sensible pour détecter les fistules durales artério-veineuses. L'ACT devrait donc être utilisée comme premier test de dépistage chez les patients qui consultent pour une HIC spontanée.

Can. J. Neurol. Sci. 2009; 36:176-180

Spontaneous intracerebral hemorrhage (ICH) is defined as non-traumatic hemorrhage into the brain parenchyma ${ }^{1}$. Spontaneous intracerebral hemorrhage accounts for $10-15 \%$ of all strokes and has a higher mortality rate than subarachnoid hemorrhage and ischemic stroke ${ }^{1}$. The etiology of ICH is classified into primary and secondary, with the former accounting for up to $88 \%$ of cases ${ }^{1}$. Primary ICH includes bleeds caused by hypertension and amyloid angiopathy, both more prevalent in the older population. Secondary causes include vascular abnormalities such as arteriovenous malformations, cavernous malformations, tumors, coagulopathy, drug use and vasculitis. Non-contrast CT head alone is insensitive and nonspecific in identifying the cause of hemorrhage ${ }^{2}$.
Digital subtraction angiography (DSA) is considered the reference standard in $\mathrm{ICH}$ where a secondary cause is suspected.

From the Division of Neuroradiology, Department of Medical Imaging, Sunnybrook Health Sciences Centre (RY, TA, RIA, AJF, SPS), Division of Neuroradiology, Department of Medical Imaging, St. Michael's Hospital ( LNdT), University of Toronto, Toronto, Ontario, Canada.

Received August 5, 2008. Final Revisions Submitted October 24, 2008 Correspondence to: Sean Symons, Neuroradiology, Department of Medical Imaging, Sunnybrook Health Sciences Centre; Department of Medical Imaging, University of Toronto, Sunnybrook Research Institute, Section of Neuroradiology, Ontario Medical Association, 2075 Bayview Avenue, Room AG31D, Toronto, Ontario, M4N 3M5, Canada. 
Digital subtraction angiography has high spatial (approximately $0.2 \mathrm{~mm}$ ) and temporal (up to 24 frames/sec) resolutions ${ }^{3}$, with the latter being important in detecting vascular malformations and shunts. The decision to undergo DSA is often based on clinical judgment weighing the risks of the procedure against that of missing a treatable lesion. The main disadvantages of DSA are its relative invasiveness, discomfort, high skill level requirement, costs and complications. The reported total risk ranges from 0 $12.2 \%$ with a $1.3 \%$ overall neurological complication rate and $0.5 \%$ for permanent neurological impairment ${ }^{3}$. There is also a $30 \%$ incidence of silent emboli ${ }^{4}$.

Computed tomographic angiography, in comparison to DSA, is a relatively non-invasive test that requires minimal additional time and cost with lower complication rates. The value of CTA in detecting aneurysms in subarachnoid hemorrhage has been well documented. However, its role in non-subarachnoid hemorrhage is not as well established. The purpose of our study is to evaluate the performance characteristics of CTA in the setting of non-subarachnoid spontaneous ICH.

\section{Materials ANd Methods}

\section{Study Cohort}

Between January 2001 and February 2007 a total of 286 patients admitted to our institution underwent both CTA and DSA for intracranial hemorrhage of all types. Fifty-five met inclusion criteria which included: 1) nontraumatic, primarily non-subarachnoid hemorrhage; and 2) CTA and DSA performed prior to any surgical intervention. Only the first CTA and DSA for each patient were included in the study. IRB approval was obtained.

\section{ImagingTechnique}

The CTAs were performed using a GE Medical Systems (Waukesha, Wisconsin, USA) Lightspeed Plus 4-slice CT or VCT 64-slice. Images were obtained from at least $\mathrm{C} 2$ to the vertex with $1.25 \mathrm{~mm}$ collimation $(120 \mathrm{kVp}, 350 \mathrm{~mA})$ on the 4slice scanner, and $0.625 \mathrm{~mm}$ on the 64 -slice scanner. Intravenous access was via an antecubital vein using an 18 or 20 gauge angiocatheter. A total of 100 to 125 ccs Omnipaque 300 or Visipaque 320 were injected at a rate of 4.0 to $4.5 \mathrm{cc} / \mathrm{second}$, with either a 17 second delay or the use of Smart Prep at the pulmonary artery. Coronal and sagittal multiplanar reformatted (MPR) images were created at $7 \mathrm{~mm}$ thickness spaced by $3 \mathrm{~mm}$. Bilateral 5 degree rotational MPRs were created at the carotid terminus. The $3 \mathrm{D}$ rendered images were created on a GE Advantage Workstation. All images were viewed on an AGFA Impax 4.5 PACS workstation.

Cerebral Angiography was performed on a Phillips uniplane neuroangiographic unit (Phillips, Erlangen, Netherlands). A standard angiographic approach was utilized: Right common femoral puncture and five french sheath. Typically a Berenstein (Boston Scientific, Mississauga, Canada) or $\mathrm{H} 1 \mathrm{H}$ catheter (Boston Scientific, Mississauga, Canada) were utilized. Selective injections were performed according to the side of the hematoma and the presence of a CTA detected abnormality. Both external and internal carotid vessels were imaged in cases where no cause was seen on CTA.

\section{Image Analysis}

Three staff neuroradiologists blinded to patient information and other imaging studies independently reviewed all CTA studies. For each study, reviewers recorded the presence or absence of a secondary cause of hemorrhage including aneurysm, arteriovenous malformation (AVM), dural arteriovenous fistula (dAVF), cavernoma and tumor. The location of hemorrhage was defined as lobar or deep according to the epicenter of the hematoma. Two staff neuroradiologists reviewed all DSAs and recorded the final vascular diagnosis. The DSAs were reviewed at least one month after the CTA review to prevent recall bias. Discrepancies between the two reviewers and the original angiogram report were resolved by evaluating follow up imaging, pathology results, surgical findings or by a consensus.

\section{RESUlts}

There were a total of 286 patients that had CTA and DSA for ICH during the study period. Of these, 231 were excluded because the cause was trauma or because the hemorrhage was primarily subarachnoid. Fifty five patients met inclusion criteria (32 males and 23 females). The median age for males and females was 49 years (range 16 to 71 ) and 50 years (range 30 to 79), respectively. The median time interval between CTA and DSA was two days with $82 \%$ performed within seven days.

The sensitivity, specificity, positive and negative predictive values and accuracy of CTA compared to DSA ranged from $88 \%$ to $97 \%$ for the three readers (Table 1). Kappa values for interobserver variability was excellent to nearly perfect $(0.78$ 0.89). False negative CTA readings occurred in single cases of AVM and Moya Moya and two of four dAVF cases. The performance characteristics of CTA were not significantly different between cases performed on 64-slice scanners compared to 4-slice scanners.

Most hemorrhages were lobar in location. Frontal lobe was most affected $(n=15)$, followed by the parietal lobe $(n=8)$ and cerebellum $(n=7)$. Basal ganglia and thalamic hemorrhages occurred in nine patients. Overall diagnostic yield for DSA was $45 \%(25 / 55)$ and $22 \%(2 / 7)$ for basal ganglia hemorrhages (Table

\begin{tabular}{lccccc}
$\begin{array}{l}\text { Table 1: Summary of the sensitivity, specificity, positive and } \\
\text { negative predictive values and accuracy for each CTA } \\
\text { reader }\end{array}$ & Sensitivity & Specificity & PPV & NPV & Accuracy \\
\hline & 88 & 90 & 88 & 90 & 89 \\
Reader 1 & 92 & 97 & 96 & 94 & 95 \\
Reader 2 & 88 & 90 & 88 & 90 & 89 \\
Reader 3 & 89 & 92 & 91 & 91 & 91 \\
Overall & & & &
\end{tabular}


Table 2: Etiology of hemorrhage based on location

\begin{tabular}{|c|c|c|c|c|c|c|c|c|}
\hline & AVM & Aneurysm & dAVF & $\begin{array}{l}\text { Moya } \\
\text { Moya }\end{array}$ & Tumour & Cavernoma & Neg & DSA Yield (\%) \\
\hline Frontal (15) & 5 & 4 & & 1 & 1 & & 4 & 67 \\
\hline Parietal (8) & 1 & & & & & & 7 & 13 \\
\hline Temporal (6) & 1 & 1 & & & 1 & 1 & 2 & 33 \\
\hline Occipital (2) & & & & & & & 2 & 0 \\
\hline Cerebellum (7) & 5 & & 1 & & & & 1 & 86 \\
\hline $\begin{array}{l}\text { Basal Ganglia } \\
\text { (9) }\end{array}$ & & 2 & & & & & 7 & 22 \\
\hline $\begin{array}{l}\text { Intraventricular } \\
\text { (5) }\end{array}$ & 1 & & 2 & & & & 2 & 60 \\
\hline Subdural (3) & & & 1 & & & & 2 & 33 \\
\hline
\end{tabular}

* Cavernoma and tumour were considered as negative DSA; AVM = arteriovenous malformation; dAVF = dural arteriovenous fistula; Total number in brackets

2). Digital subtraction angiography detected 13 AVMs (24\%), 7 aneurysms (13\%), 4 dAVFs (7\%) and 1 Moya Moya (2\%) case. The seven aneurysms presented predominantly with lobar hematomas rather than subarachnoid hemorrhage and were therefore included in the study cohort. There were three negative cases on DSA that were later found on MRI to be a cavernous malformation and two tumours.

Sixty percent of patients were older than 45 years. The DSA yield in this group was 39\% (13/33) compared to 55\%(12/22) in younger patients $(16-45$ years $)(\mathrm{p}=0.03)$.

\section{Discussion}

Although DSA is still considered the gold standard in acute $\mathrm{ICH}, \mathrm{CTA}$ is playing an increasingly important role as many are adding CTA to a non-contrast CT in the initial work-up. Noncontrast $\mathrm{CT}$ alone cannot reliably predict the presence or absence of an underlying vascular lesion (77\% sensitivity and $84 \%$ specificity reported by Halpin et $\mathrm{al}^{2}$ ). Our study shows that CTA has excellent sensitivity, specificity and accuracy (89-92\%) for determining the etiology of spontaneous ICH. Interobserver agreement was excellent to nearly perfect between our three readers (0.78 to 0.89$)$. Most of our CTAs were done on a 4 -slice CT (43/55) with more recent studies utilizing 64-slice scanners $(12 / 55)$. With increasing speed of acquisition and increased number of detectors, we anticipate increased sensitivity and specificity.

All seven acute aneurysms (Figure 1), and three incidental ones, were detected on CTA by all readers, confirming the reported high sensitivity and specificity of this modality. A metaanalysis of 78 studies found a combined $93.3 \%$ sensitivity and $87.8 \%$ specificity of $93.3 \%$ for CTA detection of intracranial aneurysms ${ }^{5}$. Computed tomographic angiography is emerging as the only pre-treatment diagnostic study in some centres ${ }^{6}$. Agid et $\mathrm{al}^{7}$ reported a $98 \%$ sensitivity and $100 \%$ specificity in aneurysm detection using 64-slice multi-detector CTA. The

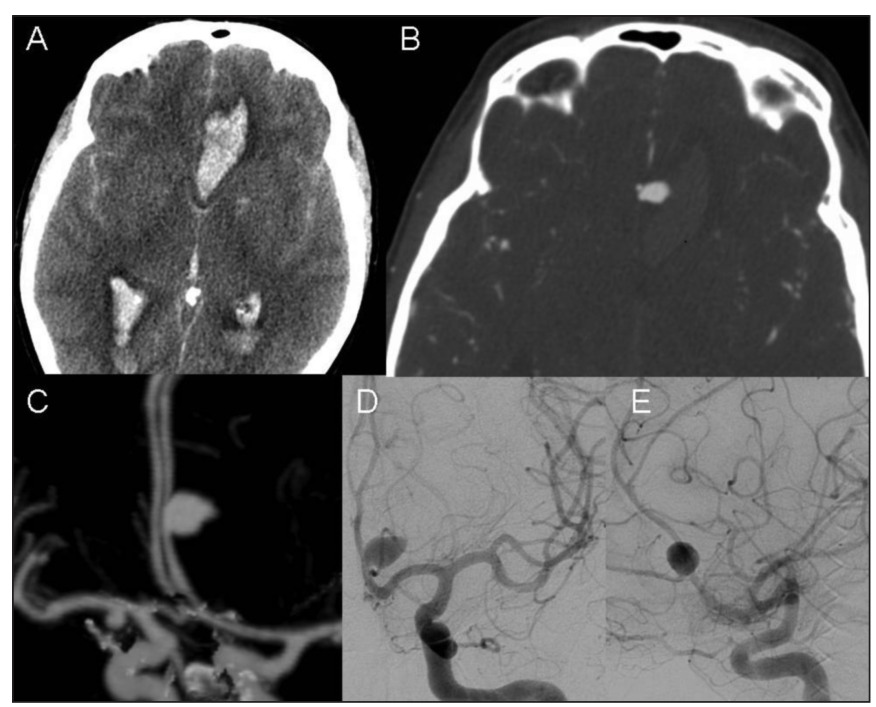

Figure 1: Pericallosal aneurysm. (A) Unenhanced CT of left frontal hematoma with subarachnoid and intraventricular blood; (B) axial CTA with $(C)$ 3-D reformatted images showing left pericallosal aneurysm. Corresponding DSA images $(D)$ frontal and $(E)$ lateral from left internal carotid artery injection. 


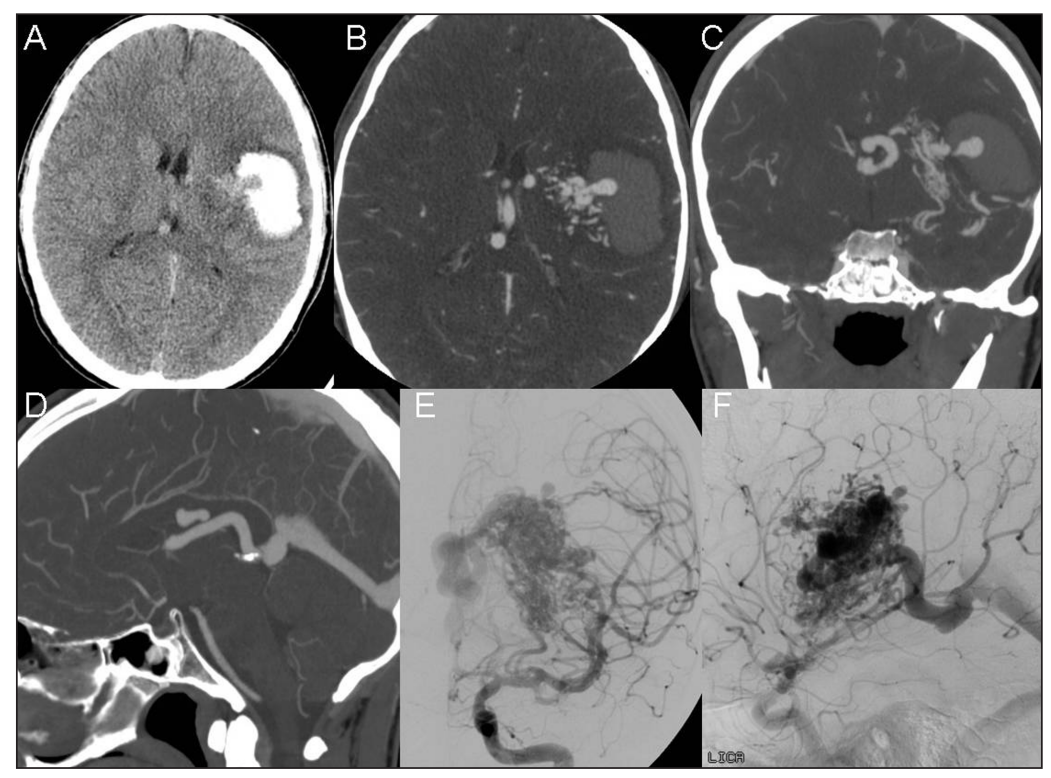

Figure 2: AVM. (A) Unenhanced CT showing left frontal hematoma. CTA (B) axial, $(C)$ coronal and $(D)$ sagittal reformatted images showing AVM. DSA $(E)$ frontal and $(F)$ lateral showing AVM draining into dilated internal cerebral veins.

smallest aneurysm detected was $1.8 \mathrm{~mm}$ and 44 of 46 aneurysms were treated based on CTA characterization alone.

Computed tomographic angiography has a high AVM detection rate in agreement with other studies (Figure 2), with only 1 of 11 missed by a single reader. Wu et al ${ }^{8}$ reported $100 \%$ specificity and sensitivity of CTA in detecting AVMs larger than $2.0 \mathrm{~cm}$ with $100 \%$ accuracy of identifying arterial feeders and $82.6 \%$ accuracy for venous drainage. Computed tomographic angiography is also highly beneficial for pretreatment stereotactic and neuro-navigation purposes, accurately localizing and measuring the nidus ${ }^{9}$. Its spatial precision limits damage to adjacent eloquent brain and reduces the volume of irradiation ${ }^{9,10}$.
Digital subtraction angiography still remains the gold standard for the precise localization and temporal filling pattern of all arterial feeders and draining veins.

A potential CTA limitation is the diagnosis of dAVFs (Figure $3)$. Half (2/4) were missed by all readers, although one was small, distant from the hemorrhage, and not likely the cause of the hemorrhage. Dural AVFs account for $10-15 \%$ of all intracranial malformations ${ }^{11}$ and the CT findings are highly variable and often non-specific. The lack of temporal resolution and peripheral location of dural feeders makes CTA identification difficult. Signs may be subtle in low grade lesions where cortical venous reflux or venous ectasia are absent. The

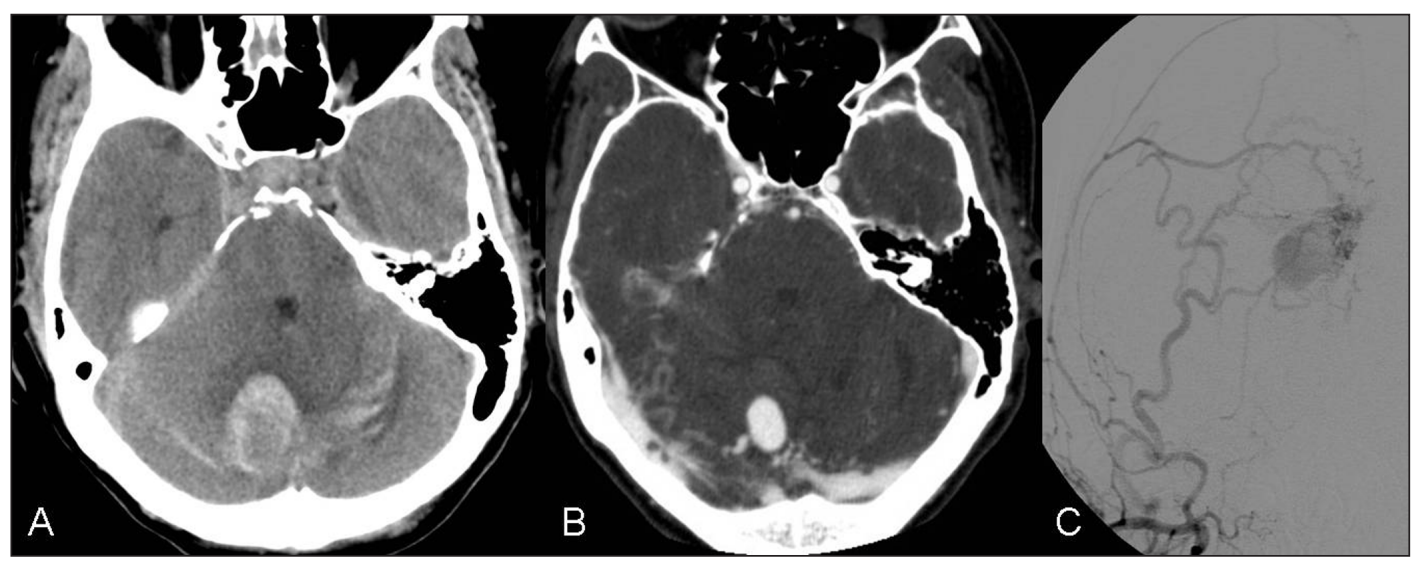

Figure 3: Dural AVF. (A) Unenhanced CT of cerebellar hematoma. (B) Axial CTA showing venous varix within hematoma and adjacent dilated serpiginous veins. (C) Frontal view of DSA from external carotid artery injection showing feeding arteries from meningeal branches. 
few reported cases of asymmetric contrast density in the dural venous sinus ${ }^{12}$, transcalvarial intraosseous channels ${ }^{13}$, and subcortical calcification related to venous congestion and hypoperfusion ${ }^{14}$ remain inadequate for screening and characterization purposes.

Magnetic resonance angiography has also been studied in the setting of $\mathrm{ICH}$. Evans et $\mathrm{al}^{15}$ reported that dynamic MRA detected all vascular abnormalities causing hemorrhage in their study. The technique allowed temporal resolution of approximately one second, however, there was spatial resolution compromise compared to time-of-flight MRA. Although MRI shows promise in acute ICH, the accessibility, availability and scan time remain drawbacks.

At our institution, CTA is part of a standard protocol including pre- and post-contrast CT for all patients presenting with spontaneous ICH. The advantages are its low risk, accessibility, and lower cost compared to DSA and MRI. A CTA facilitates expedited treatment planning in patients who may be sick or unstable. In an attempt to limit the number of patients undergoing DSA, several small series have evaluated clinical and imaging features that may predict the presence or absence of vascular lesions ${ }^{2,16,17}$. Both $\mathrm{Zhu}^{17}$ and Halpin ${ }^{2}$ found that older age (>45 years) and history of hypertension were significant independent predictors of a negative DSA. A negative CTA in our experience usually precludes any further DSA examination in older patients (greater than 60-years-old) with 'typical' hypertensive bleeds (older age, hypertension history, and basal ganglia hemorrhage). Urgent DSA is reserved for younger patients with equivocal or negative CTA or for further characterization of a detected AVM or dAVF prior to interventional treatment. The CTA highlights the vessels of interest and allows the angiographer to tailor the DSA to those vessels, thereby minimizing the vascular territories exposed to stroke risk. This allows for safer and faster DSA with less contrast use and radiation exposure.

A limitation of a retrospective study of this nature is the inability to exclude inherent selection bias. Older patients presenting with "typical" hypertensive bleeds were usually not investigated beyond a non-contrast CT. This is reflected in our somewhat high positive DSA yield (45\%). However, in general, all patients younger than 60 -years-old that present with ICH at our institution get both CTA and DSA. The purpose of this study is not to report the frequency of secondary lesions but to add to the scant data that exists in evaluating the performance of CTA compared to DSA in the context of ICH. Also, the authors intent is not to eliminate the appropriate use of DSA, but to highlight the advantages of using CTA as a first screening tool for secondary causes of ICH before deciding to proceed to DSA.

Our study has shown that CTA is an accurate modality for determining the etiology of acute spontaneous ICH compared to DSA. These results support the use of CTA in the initial diagnostic workup of ICH. This may eliminate the need for DSA when causative lesions are identified or in typical older patient hypertensive hemorrhages that are negative on CTA. The DSA may be limited to young patients with negative CTA findings or for those with vascular abnormalities treated via the endovascular route. The goal of CTA is not to replace DSA but to limit the number of patients exposed to the small but not insignificant risks associated with it. The information obtained on a positive CTA will also help the angiographer tailor the DSA study to better further evaluate the abnormality.

\section{REFERENCES}

1. Dennis MS, Burn JP, Sandercock PA, Bamford JM, Wade DT, Warlow CP. Long-term survival after first-ever stroke: the Oxfordshire Community Stroke Project. Stroke. 1993;24(6): 796-800.

2. Halpin SF, Britton JA, Byrne JV, Clifton A, Hart G, Moore A. Prospective evaluation of cerebral angiography and computed tomography in cerebral haematoma. J Neurol Neurosurg Psychiatry. 1994;57(10):1180-6.

3. Unlu E, Temizoz O, Albayram S, Genchellac H, Hamamcioglu MK, Kurt I, et al. Contrast-enhanced MR 3D angiography in the assessment of brain AVMs. Eur J Radiol. 2006;60(3):367-78.

4. Chuah KC, Stuckey SL, Berman IG. Silent embolism in diagnostic cerebral angiography: detection with diffusion weighted imaging. Australas Radiol. 2004;48(2):133-8.

5. Chappell ET, Moure FC, Good MC. Comparison of computed tomographic angiography with digital subtraction angiography in the diagnosis of cerebral aneurysms: a meta-analysis. Neurosurgery. 2003;52(3):624-31.

6. Hoh BL, Cheung AC, Rabinov JD, Pryor JC, Carter BS, Ogilvy CS. Results of a prospective protocol of computed tomographic angiography in place of catheter angiography as the only diagnostic and pretreatment planning study for cerebral aneurysms by a combined neurovascular team. Neurosurgery. 2004;54(6):1329-42.

7. Agid R, Lee SK, Willinsky RA, Farb RI, terBrugge KG. Acute subarachnoid hemorrhage: using 64-slice multidetector CT angiography to "triage" patients' treatment. Neuroradiology. 2006;48(11):787-94

8. Wu J, Chen X, Shi Y, Chen S. Noninvasive three-dimensional computed tomographic angiography in preoperative detection of intracranial arteriovenous malformations. Chin Med J (Engl). 2000;113(10):915-20.

9. Coenen VA, Dammert S, Reinges MH, Mull M, Gilsbach JM, Rohde V. Image-guided microneurosurgical management of small cerebral arteriovenous malformations: the value of navigated computed tomographic angiography. Neuroradiology. 2005;47(1):66-72.

10. Sanelli PC, Mifsud MJ, Stieg PE. Role of CT angiography in guiding management decisions of newly diagnosed and residual arteriovenous malformations. Am J Roentgenol. 2004;183(4): 1123-6.

11. Kwon BJ, Han MH, Kang HS, Chang KH. MR imaging findings of intracranial dural arteriovenous fistulas: relations with venous drainage patterns. Am J Neuroradiol. 2005;26(10):2500-7.

12. Meckel S, Lovblad KO, Abdo G, Ruiz DS, Delavelle J, Radue EW, et al. Arterialization of cerebral veins on dynamic MDCT angiography: a possible sign of a dural arteriovenous fistula. Am J Roentgnol. 2005;184(4):1313-6.

13. Alatakis S, Koulouris G, Stuckey S. CT-demonstrated transcalvarial channels diagnostic of dural arteriovenous fistula. Am J Neuroradiol. 2005;26(9):2393-6.

14. Metoki T, Mugikura S, Higano S, Ezura M, Matsumoto Y, Hirayama K, et al. Subcortical calcification on CT in dural arteriovenous fistula with cortical venous reflux. Am J Neuroradiol. 2006;27(5):1076-8.

15. Evans AL, Coley SC, Wilkinson ID, Griffiths PD. First-line investigation of acute intracerebral hemorrhage using dynamic magnetic resonance angiography. Acta Radiol. 2005;46(6): 625-30.

16. Laissy JP, Normand G, Monroc M, Duchateau C, Alibert F, Thiebot J. Spontaneous intracerebral hematomas from vascular causes. Predictive value of CT compared with angiography. Neuroradiology. 1991;33(4):291-5.

17. Zhu XL, Chan MS, Poon WS. Spontaneous intracranial hemorrhage: which patients need diagnostic cerebral angiography? A prospective study of 206 cases and review of the literature. Stroke. 1997;28(7):1406-9. 